\section{Radiographic features of the jaws and teeth in thalassaemia major}

Hazza'a AM, Al-Jamal G Dentomaxillofac Radiol 2006; 35: 283-288

Reduced haemoglobin levels may lead to changes in jaw bones.

Homozygous $\beta$-thalassaemia is an inherited haemolytic anaemia, and has been reported to have a variety of effects on the face and jaws. In a Jordanian hospital, panoramic radiographs from 19 patients of mean age 7.2 yrs and 31 of mean age 13.6 yrs with $\beta$-thalassaemia major were compared with views for 50 normal matched control subjects.

Significant differences between $\beta$-thalassaemia major patients and controls were found with respect to the prevalence of spiky roots (respectively 26\% $v$ 8\%), taurodontism (34\% $v$ $8 \%)$, large marrow space (42\% $v$ 8\%), faint lamina dura (46\% $v$ $6 \%$ ), small maxillary sinus (50\% $v 4 \%$ ), borders of the inferior dental canal (18\% v 92\%), and mean cortical bone thickness $(2.5$ $v 4.2 \mathrm{~mm}$ ). Mean crown and root length were also significantly less in $\beta$-thalassaemia patients. The authors consider that the radiographic signs may help corroborate diagnosis of these patients.

doi:10.1038/sj.bdj.4814308

\section{ORAL HYGIENE; RESPIRATORY CONDITIONS}

\section{Systematic review of the association}

between respiratory diseases and oral health

Azarpazooh A, Leake JL J Periodonto/ 2006; 77: 1465-1482

There is evidence that oral disinfection may reduce pneumonia.

Currently there is renewed interest in whether oral health affects other disease processes. This study examined evidence on chronic obstructive pulmonary disease (COPD) and pneumonia. From a total of 74 relevant titles, these reviewers identified 19 papers of importance.

In 4 prospective cohort studies and 1 case-control study, pneumonia was associated with oral health. In 2 case-control and 2 cross-sectional studies, COPD was associated with oral health. In 10 clinical trials, 3 of which were not randomised, reduction of plaque and oral microflora by professional care, chlorhexidine or non-absorbable antibiotics led to improved respiratory health.

Evidence of a causal relationship between oral and respiratory diseases was not apparent, but reduced oral microbial load appears to benefit patients at risk of pneumonia.
A thermoplastic mandibular advancement device for the management of non-apnoeic snoring: a randomized controlled trial

Cooke ME, Battagel JM Eur J Orthod 2006; 28: 327-338

The device proved useful in $2 / 3$ of subjects.

Snoring is a common cause of social disharmony. Most snorers do not have obstructive sleep apnoea, which has a risk of complications. Various methods have been used to treat snoring, ranging from weight loss to surgery. Custom-made appliances are relatively inexpensive and some can be made at the chairside. In this study, 27 patients (23 completed the study) who had a diagnosis of nonapnoeic snoring with a tongue base contribution to it entered a randomized cross-over trial of wearing an appliance with (active position) and without (placebo) mandibular advancement. The 2 study periods were each of 4-6 weeks, separated by a 1 day washout.

Median snores with advancement reduced from 398 to 17 per hour. Oxygen saturation also improved. A 9 dimension quality of life questionnaire revealed no significant differences between not wearing an appliance and the 2 appliance positions. Bulk and dry mouth were the main drawbacks. However, partners reported improvements in their sleep quality and reductions in daytime tiredness. The authors point out that the appliance used has the advantage of being adjustable.

doi:10.1038/sj.bdj.4814309

\section{ORAL PATHOLOGY}

\section{An analysis of oral and maxillofacial pathology found in adults over a 30-year period}

Jones AV, Franklin CD J Oral Pathol Med 2006; 35: 392-401

In a UK hospital, $5 \%$ of these specimens proved malignant.

From 1973 to 2002, 53,666 specimens were received in a UK department of oral pathology. After excluding those for which data were incomplete, for subjects under 16 yrs and for recurrence (mostly squamous cell carcinoma), 44,007 cases were included in the study.

The principal diagnoses were: mucosal pathology (36\%), odontogenic cysts (14\%), tooth pathology (10\%), salivary gland pathology (6\%). Benign tumours accounted for 5.6\% and malignant tumours for 5.4\%.

The authors conclude that most diagnoses were benign and required no further surgery, but there were around 10\% which required long-term management (such as lichen planus or keratocysts) and about 5\% required major malignancy surgery.

doi:10.1038/sj.bdj.4814311 